\title{
PID electro-hydraulic cylinder force tracking system with friction compensation
}

\author{
Weerapong Chanbua $^{1 *}$, and Unnat Pinsopon ${ }^{1}$ \\ ${ }^{1}$ Department of Mechanical Engineering, Faculty of Engineering, King Mongkut's Institute of TechnologyLadkrabang, \\ Bangkok, Thailand
}

\begin{abstract}
Friction can be found in all mechanical systems, and is the cause of control tracking error. In this article, LuGre friction model for symmetric hydraulic cylinder was studied and obtained experimentally. The estimated friction force was applied for reference force compensation. Force tracking performances of PID force control systems with and without friction compensation were tested and compared. Control system with friction compensation outperformed one without in all cases of tracking tests. The best result was found in the square force tracking tests, with an average error at maximum compression force of $86.105 \mathrm{~N}$ in the case of with friction compensation compared with error of $511.996 \mathrm{~N}$ in the case without. However, the estimated friction achieved in the study was noisy. This is due to the use of noisy numerically differentiated velocity signal in the friction estimation procedure.
\end{abstract}

\section{INTRODUCTION}

Electro-hydraulic control systems could be applied for position control, velocity control or force control. Electrohydraulic force control systems are used various industrial applications such as press machine, earth moving equipment [1] and flight simulator [2]. Hydraulic cylinder is the main actuator used in force control applications. Friction occurs in cylinder at the contact areas between piston seal and cylinder barrel, and between piston rod seal and wiper. Friction depends on surface contact, temperature, lubrication and contact velocities. Friction always occurs in mechanical systems, and leads to control tracking error. Friction compensation is one of most popular method to solve the problem. Various friction models were reviewed in great details in [3] and [4]. The prediction ability of four friction models were compared in experiments [4], and LuGre friction model was found to be the all-round best predictor.

In this paper, an electro-hydraulic cylinder force control system was constructed. The force feedback signal used in the control scheme was calculated using the value of oil pressures at both sides of cylinder. LuGre friction model was used to predict cylinder friction force. The predicted friction would be used as force reference compensation in the PID control scheme. Force tracking performances of the PID controller with and without friction compensation would be tested and compared.

\section{FRICTION MODEL}

General steady state friction characteristics are presented in figure 1 , whereas friction is shown as a velocity function. The Coulomb and the stiction friction forces are constant and independent of velocity. The viscous friction force is caused by the viscosity of lubricants, and increases with velocity. The Stribeck effect is the phenomenon at low velocity region that friction decreases with the increasing of velocity.

LuGre friction model is a widely used dynamic friction model which describes the microscopic deflection between the contact surfaces in terms of a damping-spring behavior [3]. The LuGre model described the deflection of elastic bristles which represents the asperities between two contacting surfaces. The average deflection of the bristles is denoted by $z$ and the deflection rate of $z$ is modeled by equation 1 . The average deflection of the bristles is related to the friction force as shown in equation 2.

$$
\begin{gathered}
\frac{d z}{d t}=v-\frac{\sigma_{0}}{g(v)}|v| \\
F_{\text {friction }}=\sigma_{0} z+\sigma_{1} \frac{d z}{d t}+\sigma_{2} v
\end{gathered}
$$

Where $v$ is velocity between contact surfaces, $\sigma_{0}$ is the average stiffness of the bristles, $\sigma_{1}$ is the damping coefficient and $\sigma_{2}$ is viscous friction coefficient. At steady state, $\frac{d z}{d t}$ is zero, and $\sigma_{0} z$ is equal to $g(v)$. Therefore the steady state friction is calculated by

$$
F_{\text {friction ss }}=g(v)+\sigma_{2} v
$$

\footnotetext{
* Corresponding author : weerapong.kmitl@,gmail.com
} 
$g(v)=F_{\text {coulomb }}+\left(F_{\text {stiction }}-F_{\text {coulomb }}\right) e^{-\left|\frac{v}{v_{\text {stribeck }}}\right|^{n}}$

Where $F_{\text {coulomb }}$ is the coulomb friction force, $F_{\text {stiction }}$ is the stiction friction force, $v_{\text {stribeck }}$ is the stribeck velocity, $n$ is the exponent and

$F_{\text {coulomb }}+\left(F_{\text {stiction }}-F_{\text {coulomb }}\right) e^{-\left|\frac{v}{v_{\text {stribeck }}}\right|^{n} \text { is the stribeck }}$

friction. The values of these parameters are to be determined experimentally.

The steady state friction can be expressed by

$$
\begin{aligned}
F_{\text {friction ss }}=F_{\text {coulomb }}+ & \left(F_{\text {stiction }}-F_{\text {coulomb }}\right) e^{-\left|\frac{v}{v_{\text {stribeck }}}\right|^{n}} \\
& +\sigma_{2} v
\end{aligned}
$$

The steady state friction force calculated by equation 5 could well describe the general steady state friction characteristics (figure 1).

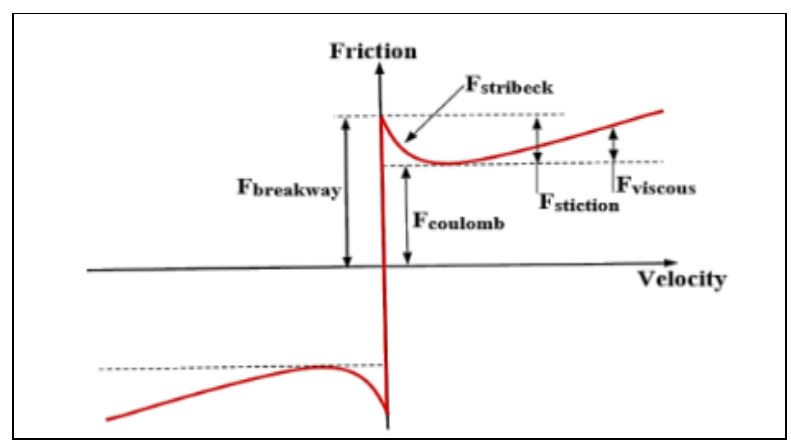

Fig. 1. Steady state friction characteristics.

\section{EXPERIMENTAL APPARATUS AND METHODS}

\subsection{Experimental apparatus}

Figure 2 shows the electro-hydraulic force control experimental apparatus. The cylinder used is the symmetric double-rod type with $0.04 \mathrm{~m}$ bore diameter, $0.028 \mathrm{~m}$ rod diameter and $0.50 \mathrm{~m}$ stroke. The motion of the cylinder was controlled by a proportional valve which receives oil flow from a gear pump. Pressure transducers were installed at both sides of the cylinder. Since direct force measurements are usually impractical in actual industrial applications, feedback cylinder force was calculated based on the cylinder pressures (equation 6). A load cell is also installed at the end of the cylinder rod in the setup. However, the force signal measured by the load cell is used as reference only. A draw wire potentiometer was used to measure piston displacement which would be numerically differentiated to obtain cylinder velocity. A compression spring was used as the simulated cylinder load.

$$
F_{\text {cylinder }}=\left(P_{1}-P_{2}\right) A
$$

Where $F_{\text {cylinder }}$ is cylinder force, $P_{1}$ and $P_{2}$ are oil pressures in hydraulic cylinder and $A$ is piston area.

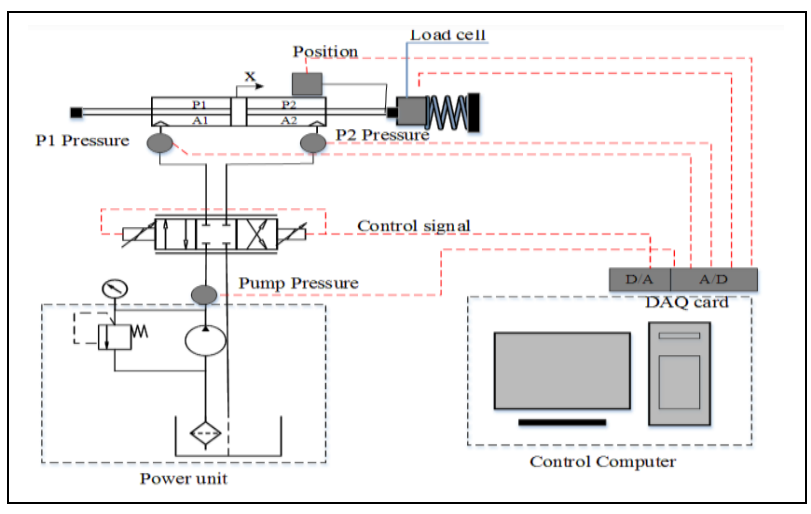

Fig. 2. Electro-hydraulic force control experimental apparatus.

\subsection{Experimental methods}

The experiments are divided in two sections. First, the friction model parameters would be identified, and the steady state LuGre friction model according to equation 5 will be constructed. Second, PID force tracking controls with and without friction compensation would be tested and compared.

\subsubsection{Friction parameters identification}

Friction force in hydraulic cylinder is obtained from force balance of the cylinder piston.

$$
F_{\text {friction }}=\left(P_{1}-P_{2}\right) A-m
$$

Where $F_{\text {friction }}$ is friction force, $m$ is piston mass, is piston acceleration. Various constant commands were sent to the proportional valve, frictions at various velocities were then calculated by equation 6 and recorded. Generalized reduced gradient nonlinear (GRG) algorithm was used for fitting the recorded frictions into the LuGre steady state friction model (equation 5) in order to obtain the values of friction parameters.

\subsubsection{Force tracking control}

Figure 3 shows the diagram of PID force control system. In addition to the cylinder force feedback, velocity signal was also fed back to LuGre friction model. The output from LuGre friction model, the estimated friction, would be used as compensation to the reference force signal. The tracking performances of PID force tracking with and without friction compensation would be tested and compared.

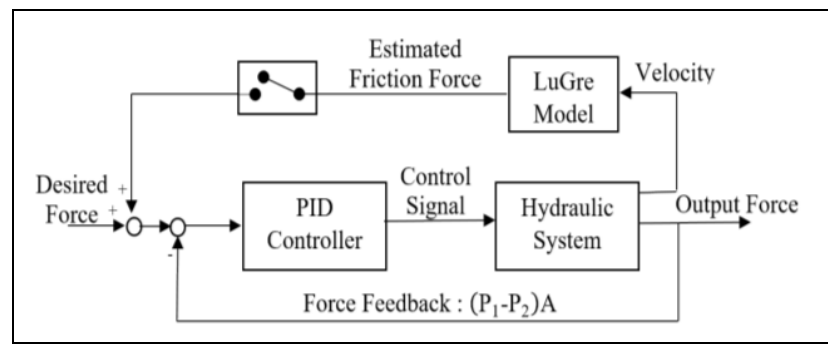

Fig. 3. Block diagram of PID force control with friction compensation. 


\section{EXPERIMENTAL RESULT}

\subsection{Friction parameters identification}

Figure 4 shows the comparison between the measured steady state friction (obtained from equation 7) and the estimated friction (obtained from equation 5). The LuGre model parameters were obtained by GRG algorithm. The friction parameters were obtained separately according to the direction of motion. Equation 8 described the friction for the positive (right hand side) motion, while equation 9 described the friction for the negative motion. Equations 8 and 9 were coded in the friction model of the control scheme.

$$
\begin{aligned}
F_{\text {friction ss,extend }}= & 32.37+440.1163 e^{-\left|\frac{v}{0.000000111}\right|} \\
& +1068.905 v
\end{aligned}
$$

$F_{\text {friction ss, retract }}=(-204.351)+\left.(-307.096) e^{-\mid \frac{v}{-0.0128}}\right|^{1.215129}$

$$
+581.397 v
$$

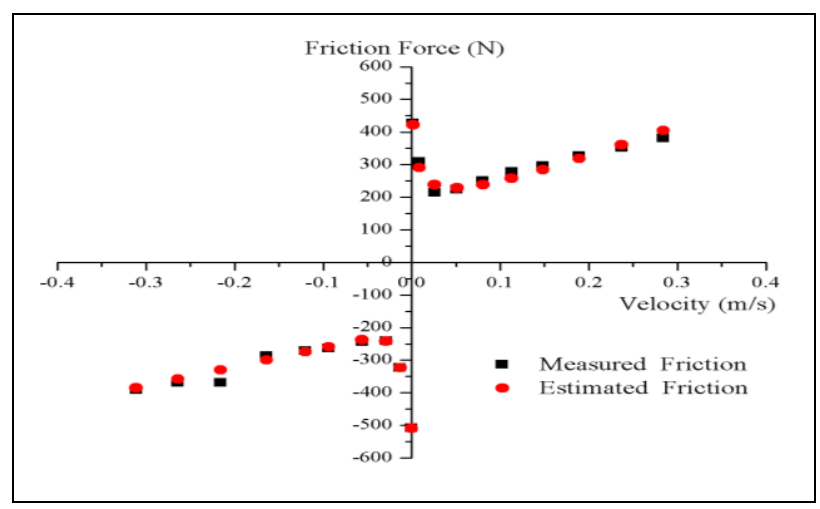

Fig. 4. Comparison of measured frinction and estimated friction

\subsection{Experimental Results and Discussion}

The force tracking performances were done with two type force reference signals, sinusoidal signal and square signal. The same PID controller gains were used in the control schemes with and without friction compensation. The PID gains were first tuned with Ziegler-Nichols method, and finely tuned later in experiments. The desired sinusoidal and square force signals had minimum and maximum amplitudes of $500 \mathrm{~N}$ and $3,500 \mathrm{~N}$, both at a frequency of 0.2 $\mathrm{Hz}$.

Figure 5 shows the tracking performances of the PID controller systems with sinusoidal force reference signal. As mentioned earlier, the feedback force signals used in both control schemes were calculated using the cylinder pressures. In order to realize the actual forces on the load, the tracking forces of both control schemes shown in figure 6 were directly obtained from measurement of the load cell. It can be seen that the control system with friction compensation performed better when the piston moved in the positive direction. The average tracking errors at maximum compression force are $264.826 \mathrm{~N}$ and $569.342 \mathrm{~N}$ for the controllers with and without friction compensation, respectively. Figure 6 shows the estimated friction force and the calculated friction force obtained from the sinusoidal force tracking test. The estimated friction force signal was noisy in zones a and b (figure 6). The cylinder velocity signal was obtained by numerically differentiation of position signal, and was noisy at the low velocity regions of zones $\mathrm{a}$ and $\mathrm{b}$. This leads to noisy estimated friction force according to equation 5. The control actions of the controllers with and without friction compensation are shown in figure 7. The control actions of two cases resembled each other for almost all of the time, except at the times about 4, 9, 13, and 18 seconds (figure 7). The control action of the controller with friction compensation surged at higher values at those instants time. Those are the times that cylinder switched the direction of motion from negative to positive direction. The surged control action would give the cylinder a kick start at the instant of direction switch, and overcame the breakaway friction. This resulted in smaller tracking error at maximum compression force. The RMS errors of sinusoidal force tracking are 371.386 $\mathrm{N}$ and $457.737 \mathrm{~N}$ for the controllers with and without friction compensation, respectively. The RMS errors as well as average errors at maximum compression forces are also shown in in table 1.

Figures 8 to 10 show the experimental results from square force tracking tests. The controller with friction compensation outperformed the controller without friction compensation, with an average error at maximum compression force of $86.105 \mathrm{~N}$ compared with an average error of $511.996 \mathrm{~N}$ in the case of without friction compensation. The control action of the controller with friction compensation surged at higher values compared with the controller without friction compensation at the times 5, 10 and 15 seconds (figure 10). This would give the cylinder a kick start at the motion starting instant, the same action that could be observed in the sinusoidal force tracking tests. The cylinder velocity in square force tracking tests was low about zero at almost all of the time. Therefore, the numerically differentiated velocity signal was noisy almost all of the time, and so was the estimated friction force (figure 9). The RMS tracking errors in the cases of with and without friction compensation are $809.743 \mathrm{~N}$ and $976.401 \mathrm{~N}$, respectively. For these square force tracking tests, the maximum compression lasted only half of the period of the reference command. Had the maximum compression lasted longer, the difference in RMS errors between two cases would be larger.

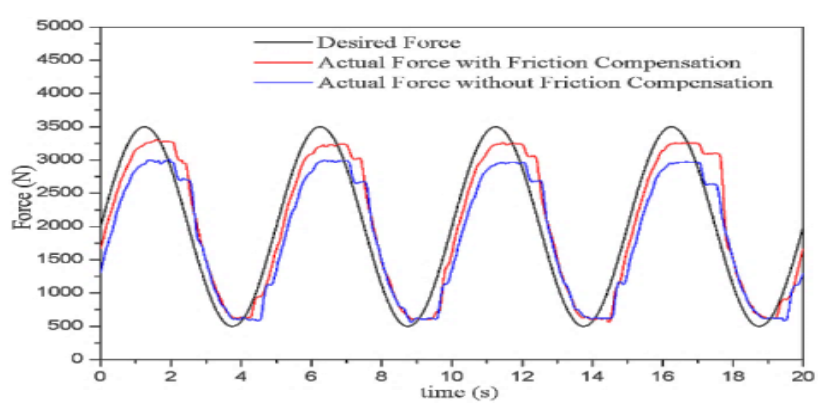

Fig. 5. Force tracking control performances of sinusoidal force tracking tests. 


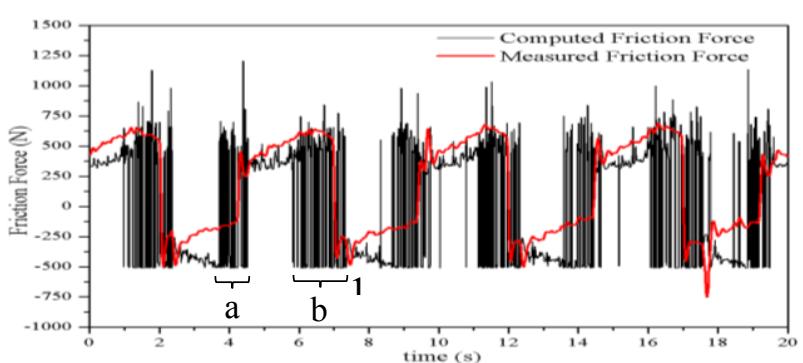

Fig. 6. Comparison of measured and computed friction forces in sinusoidal force tracking tests.

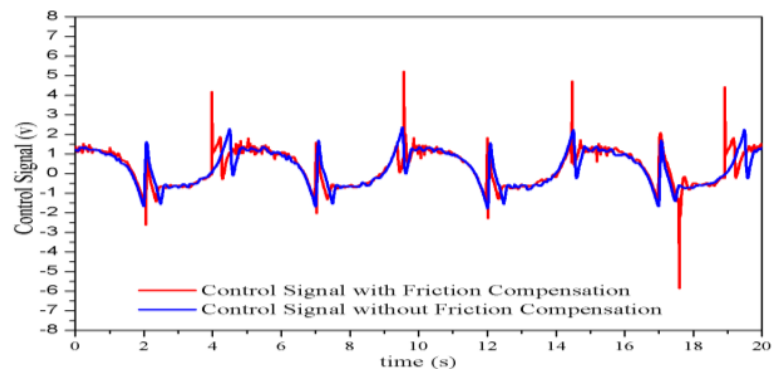

Fig. 7. Comparison of control signals in sinusoidal force tracking tests.

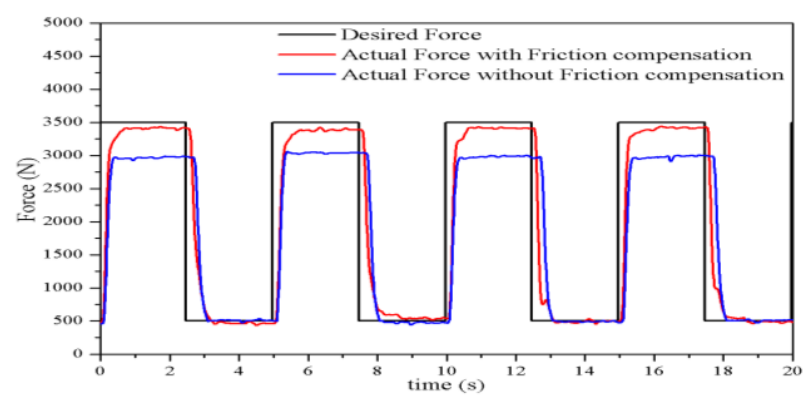

Fig. 8. Force tracking control performances of square force signal tracking tests.

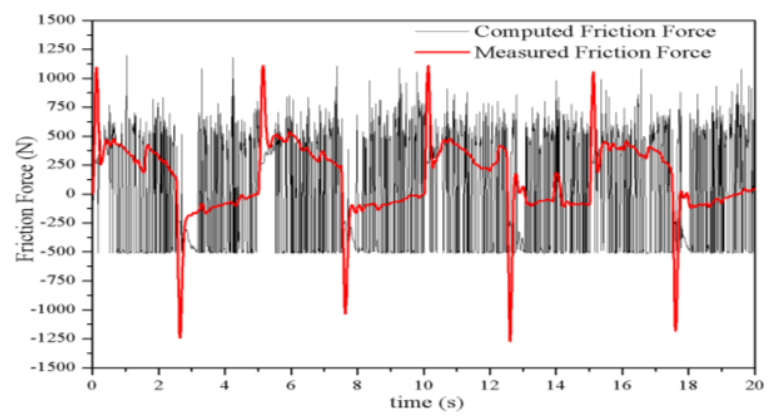

Fig. 9. Comparison of measured and computed friction forces in square force tracking tests.

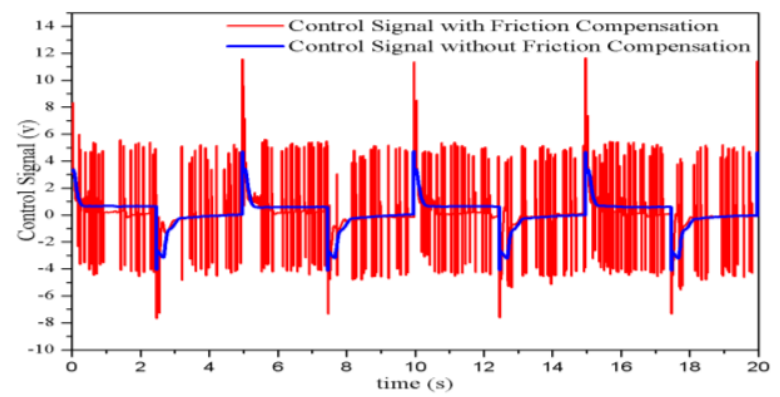

Fig. 10. Comparison of control signals in square force tracking tests.
Table 1. RMS error and error at maximum force

\begin{tabular}{|c|c|c|c|c|}
\hline \multirow{2}{*}{$\begin{array}{c}\text { Reference } \\
\text { Command }\end{array}$} & \multicolumn{2}{|c|}{$\begin{array}{c}\text { Without friction } \\
\text { compensation }\end{array}$} & \multicolumn{2}{c|}{$\begin{array}{c}\text { With friction } \\
\text { compensation }\end{array}$} \\
\cline { 2 - 5 } & $\begin{array}{c}\text { RMS } \\
\text { error (N) }\end{array}$ & $\begin{array}{c}\text { Error at } \\
\text { maximum } \\
\text { force (N) }\end{array}$ & $\begin{array}{c}\text { RMS } \\
\text { error (N) }\end{array}$ & $\begin{array}{c}\text { Error at } \\
\text { maximum } \\
\text { force (N) }\end{array}$ \\
\hline Sinusoidal & 457.737 & 569.342 & 371.386 & 264.826 \\
\hline Square & 976.401 & 511.996 & 809.743 & 86.105 \\
\hline
\end{tabular}

\section{CONCLUSIONS}

In this paper, LuGre friction model in symmetric hydraulic cylinder was experimentally constructed. The estimated friction force was applied as compensation to the reference force signal. Tracking performances of PID force controllers with and without friction estimation were tested and compared. Feedback force signal used in both control schemes were calculated using the cylinder pressures.The control system friction compensation achieved better tracking performances. Friction compensation could give cylinder a kick start and overcame the breakaway friction force. This resulted in much lower tracking errors at maximum compression force in both sinusoidal and square force tracking. The estimated friction force obtained in this study was noisy due to the use of numerical differentiated velocity signal. Had a higher performance velocity filter been used, better estimated friction force would had been obtained, better tracking performance could had been achieved.

\section{Reference}

1. Q. P. Ha, Q. H. Nguyen, D. C. Rye,H. F. DurrantWhyte, Force and Position Control for Electrohydraulic systems of a Robotic Excavator, Proceedings of the 39th IEEE Conference on Decision and Control, Vol. 5, 5224-5229 (2000)

2. D. Q. Truong, K. K. Ahn, Force Control for Hydraulic Load Simulator using Self-Tuning Grey Predictor - fuzzy PID, International Federation of Automatic Control, Mechatronics 19, 233-246 (2009)

3. H.Olsson, K.J.Åström, Canudas de Wit M.Gäfvert, P.Lischinsky, Friction Models and Friction Compensation, European Journal of Control, Vol. 4, Issue 3, 176-195 (1998)

4. Y. Sun, Tao Chen, C. Q. Wu, C. Shafai, Comparison of Four Friction Models: Feature Prediction, Journal of Computational and Nonlinear Dynamics, Vol. 11, $1-10(2016)$ 